\title{
Analysis of the difference in characteristics of patients and IVF cycles and the parameters of in vitro embryo culture between normal individuals with high and low blastocyst formation rate
}

\section{Chun-mei Yu}

Changzhou Women and Children's Hospital

\section{Xiu-liang Dai}

Changzhou Women and Children's Hospital

\section{yu-feng Wang}

Changzhou Women and Children's Hospital

Li Chen ( $\nabla$ czrcchenli@126.com )

Changzhou Women and Children's Hospital

\section{Research}

Keywords: Useful blastocyst formation, normal patients, individual characteristics,basic LH, ovarian response, in vitro embryo culture

Posted Date: March 13th, 2020

DOI: https://doi.org/10.21203/rs.3.rs-17214/v1

License: (c) (1) This work is licensed under a Creative Commons Attribution 4.0 International License. Read Full License 


\section{Abstract}

Background: The main purpose of this study is to analyze the possible factors that were related with high useful blastocyst formatio rate in normal patients.

Methods: This was a retrospective cohort study included 706 normal patients(excluding PCOS, DOR, endometriosis and other special diseases),according to the useful blastocyst in vitro formation

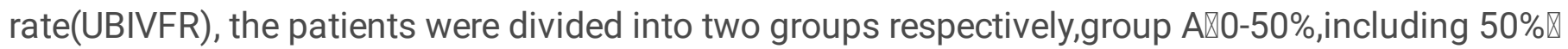
and group B囚50\%-100\%).

Results: There were no significant difference in patient ages between the two groups,compared with group $A$, the level of basic $\mathrm{LH}(\mathrm{bLH})$ was significantly increased. The clincal data showed that ,compared with group $A$, the total $\mathrm{G}$ dose and the number of dominant follicles, oocytes retrieved冈MII mature oocytes, normal fertilized oocytes rate,high-quality embryos rate and FET clinical pregnancy rate were significantly higher than group $B$.

Conclusion:Our study is to investigate the useful blastocyst formation rate in relation to bLH levels in IVF/ICSI cycles, showing that high bLH is associated with higher ovarian response and higher UBIVFR.AMH,bFSH and AFC which were defined as ovarian reserve and ovarian response are seem not associated with the useful blastocyst formation. Key words:Useful blastocyst formation; normal patients; individual characteristics; basic LH; ovarian response; in vitro embryo culture

\section{Background}

ART is efficient technology (sometimes it is the only way) that allows infertitle couples have a baby.Recent years the ART is developing at a high speed,expending the in vitro culture time from 3 days to $5 / 6$ days is a big progress(1).Day 3 embryo was identified as cleavage embryo while Day5/6 embryo was blastocyst stage embryo (a later developmental stage)(2).Not all the zygots have the livebirthpotential,in fact,sometimes only a small number of zygots can lead a livebirth. Therefore,how to choose the livebirth-potential embryo is very important.It is believed that blastocyst culture will contribute to embyo selection. This is because of some of embryos with limited developmental will be eliminated during expended in vitro culture.Numerous studies have reported that transfer of blastocyst resulted in increasing clinical pregnant and live birth rate and reduced abortion rate $(3,4)$.Due to this, many reproductive centre have developed "all blastocyst culture strategy".

Couples produce more health embryos have more chance to have a baby.However,it is difficult to distinguish"good" embryos from total cleavage embryos that they may have the same morphology score(5).Blastcyst culture somewhat may improve this situation.In another word ,more useful blastocyst obtained from a couple means more chance they have to have a baby.Therefore,the useful blastocyst in vitro formation capacity(UBIVFC) for any couples matters. We thought that the useful blastocyst formation rate (UBIVFR) based on a certain number of day 3 embryos for further culture can reflect 
UBIVFC.Analysis of the difference in baseline characteristics of patients and IVF cycles between patient with high and low UBIVFC and to guide and improve the therapeutic strategy.

In the present study,from March 2017 to september 2018 in our center, women undergoing IVF/ICSI reginment for the treatment of infertility were recruited, excluding PCOS, DOR, endometriosis and other special diseases.

\section{Methods}

\section{Patients}

A retrospective cohort study was conducted at the Changzhou maternal and child health care hospital. From March 2017 to september 2018,women undergoing IVF reginment for the treatment of infertility were recruited. The study protocol was approved by the Ethics Committee of the Changzhou maternal and child health care hospital. It was conducted based on the Declaration of Helsinki for medical research. All participants provided informed consent after approving for infertility treatments and routine IVF procedures.

Since two D3 high-quality cleavage embryos were transplanted or frozen, more than two remaining embryos were used for expending culture, only patients with a chance to reach blastocyst stage were included as defined by $\geq 5$ normal fertilized oocytes.Polycystic ovary syndrome(PCOS),ovarian function decline(DOR) endometriosis and other diseases were excluded from the study population.The remaining patients were our subjects and defined as normal individuals.

\section{Blastocyst score}

All our embryologists are trained and use this classification of Gardner and Schoolcrafton $₫ 1999$ \to classify the blastocysts in daily clinical practice (7). Briefly, according to blastocysts degree of expansion and hatching status, blastocysts were given a numerical score from 1 to 6 , as follows: 1 , An early blastocyst whose blastocoel is less than half the volume of the embryo; 2 , A blastocyst in which the blastocoel is half or more of the volume of the embryo; 3 , The blastocoel is filled to the full embryo; 4 , an expanded blastocyst with a blastocoel volume larger than that of the early embryo, with a thinning zona; 5 , a hatching blastocyst with the trophectoderm starting to protrude though the zona; and 6, An incubated blastocyst in which the blastocyst has completely escaped from the zona.For blastocysts graded as 3-6 (full blastocysts onward), the development of the inner cell mass was assessed as follows: A,many cells,tightly packed; $B$, several cells,loosely grouped; or $C$, very few cells. The trophectoderm was assessed as follows: A, many cells forming a cohesive epithelium; B, few cells forming a loose epithelium; or C, very few large cells. According to the scoring rules,transferable blastocyst was analysed, not including $4 \mathrm{CC}, 5 \mathrm{CC}$ and $6 \mathrm{CC}$. There was two embryologists with the six years'scoring experience to grade the blastocysts simultaneously in order to reduce the intra-variability and inter-variability. 
According to the useful blastocyst formation rate, the patients were divided into two groups respectively,group $A(0-50 \%$,including $50 \%)$ and group $B \otimes 50 \%-100 \% \bigotimes$.

\section{Serum hormone analysis}

Serum were collected on menstrual cycle day 3 and the trigger day respectively. Hormones including $\mathrm{AMH}, \mathrm{FSH}, \mathrm{LH}, \mathrm{E} 2$ and progesterone levels were measured by chemiluminescence (Abbott Biologicals B.V., The Netherlands). The lower limits of sensitivity were as follows: $\mathrm{FSH}=0.06 \mathrm{mlU} / \mathrm{ml}, \mathrm{LH}=0.09 \mathrm{mlU} / \mathrm{ml}$, $\mathrm{E} 2=10 \mathrm{pg} / \mathrm{ml}$ and $\mathrm{P}=0.1 \mathrm{ng} / \mathrm{ml}$.

\section{Statistical Analyses}

All statistical analyses were performed with the use of Graphpad prism 5(GraphPad Software Inc., San Diego, CA, USA ). These data found to be normally distributed were presented as the mean \pm standard deviation (SD), and were tested to a Students t-test. Those data that were not normally distributed were presented as the mean (interquartile range), and were analyzed using a MannWhitney U-test. Categorical variables were expressed as a frequency (percentage), and analyzed using a Chi-square test. A value of $P$ $<0.05$ was considered to indicate statistical significance.

\section{Results}

\section{Patient characteristics}

No difference in age, and basal FSH, E2, P ,T value was observed between the two groups.Similarly,type of infertility(n),patient diagnoses,ovulation promotion program, cycle number and methods of insemination were not significantly different between two groups. However,compared with the group $A$, the basal luteinizing hormone (bLH) in group B was significantly increased .(Table 1).

\section{Follicle development, oocyte performance, and clinical outcomes}

The clinical data in two groups were described in the table 2.Compared with the group A,in group B the total $\mathrm{Gn}$ dose and the number of dominant follicles (diameter larger than $14 \mathrm{~mm}$ ), oocytes retrieved $\llbracket \mathrm{MII}$ mature oocytes, normal fertilized oocytes rate, ,high-grade(grade I and grade II) embryos rate and FET 
clinical pregnancy rate were significantly higher.The FSH levels were significantly lower》p $<0.05$ \and E2 levels were significantly higher $\ \mathrm{p}<0.05 \rrbracket$ on the HCG trigger day. There were no statistical differences in LH levels and $\mathrm{P}$ levels.

\section{The subgroup analyses by age,BMl,bFSH and AFC between two groups}

Table 3 presents that the subgroup analyses by age,BMI,bFSH and AFC were conducted between two

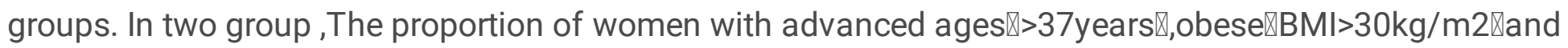

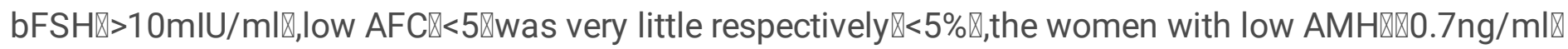
was zero.There was no statistical difference in the proportion of different subgroups of the same index.

\section{Discussion}

Our study found that the basic LH levels of patients with high UBIVFR were signifcantly higher than the patients with low UBIVFR $(5.31 \pm 3.19 \& 5.68 \pm 2.83)(p<0.001)$,suggested bLH levels may be associated with the blastocyst formation. The influence of $\mathrm{LH}$ on follicle maturation in vitro showed that follicle survival rate was signifificantly higher in medium supplemented with $\mathrm{LH}$ than without (8). Moreover, LH bioactivity had an effect on antral cavity formation(9). Some scholars have found that the threshold of $\mathrm{LH}$ is 1.2 in patients with hypogonadotropic hypogonadism $(\mathrm{HH})[10]$. If the threshold of $\mathrm{LH}$ is lower than this, the level of E2 will be low, which will affect the development of follicles.Other literature shows that women with $\mathrm{LH}$ less than $10 \mathrm{IU} / \mathrm{L}$ have higher pregnancy rate and lower abortion rate than those with basic LH greater than $10 \mathrm{IU} / \mathrm{L}[11,12]$. The basic LH of patients in our study was less than $10(5.31 \pm 3.19$ \&5.68 \pm 2.83 ), it can be inferred that high basic LH in a certain range is better for the blastocyst formation and it may be specifical reflective of the" LH window"[13] theory of follicular development.

The data of our study showed that the dosage of $\mathrm{Gn}$ in patients with UBIVFR is significantly more than in patients with low UBIVFR.Otherwise, the number of oocytes retrieved is significantly less than that in patients with low UBIVFR.Some study confirmed the "ovarian sensitivity index "(OSI,the ratio between the oocyte yield and the total dose of Gn defined ovarian response which is essential to the performance of assisted reproduction by IVF/ICSI(14).Previous study has confirmed an excellent correlation between the parameters of ovarian response and ISO(15).Calculated that our results suggested ovarian response of patients with high UBIVFR better than the patients with low UBIVFR and it also confirmed that patients in our study are normal population, because the range of ISO with normal response is $1.697 / \mathrm{IU}$ 10.07/IU.Clinical outcome data show that the number of MII mature oocytes, normal fertilized oocytes, high-grade(grade I and grade II) embryos and FET clinical pregnancy rate in patients with high UBIVFR was significantly higher and suggedted that the ovarian response may be assosiated with blastocyst formation.

In clinical practice,we often use $\mathrm{AMH}, \mathrm{bFSH}$ and $\mathrm{AFC}$ as calculators of ovarian reserve and ovarian response.Previous study have indicated that elevated bFSH levels(>10 IU/L)[16],low level of $\mathrm{AMH}(<$ $0.7 \mathrm{ng} / \mathrm{ml})[17]$ and $\operatorname{AFC}(<5)[18]$ reduction correlated with poor response and poor pregnancy 
outcomes,but there are no significantly difference in three indexes in our study $(p>0.05)$. On the one hand,due to the limited criteria for enrollment,the proportion of the patients with elevated bFSH levels,low level of $\mathrm{AMH}$ and $\mathrm{AFC}$ is lower and there is no difference, patients with $\mathrm{AMH}(<0.7 \mathrm{ng} / \mathrm{ml}) \mathrm{make}$ up zero in two group. On the other hand,we speculated that ovarian reserve markers serve as a proxy for follicle quantity but are considered poor predictors of ovarian response in normal patients.Some recent studies have confirmed this thought,the availability of records from 981 women 30-44 years of age without a history of infertility who trying to conceive for 3 months and 8241 patients $21-44$ years of age undering fresh IVF cycles,do not support the use of bFSH,AMH and AFC to assess ovarian response,oocyte quality and embryo development [19][20].The present results suggest that bFSH,AMH,and AFC is not associated with the ovarian response and the useful blatocyst formation of normal patients.

It proved that age is still remains the best predictor to oocyte quality and embryo development[21].Aged oocytes exhibit increased chromosomal abnormalities and dysfunction of organelles, both of which factor affected oocyte quality[22][23]. But in our study there is no difference in the age of different UBIVFR, the reason is that it is possible the average age in the two group was lower than 35(30.1 \pm $3.62 \& 29.8 \pm 3.47$ ). The results of age subgroup in the two groups showed that most of the patients were younger than 35 years, and only those older than 35 years accounted for $12.9 \%(308 / 354)$ and $9.4 \%$ (320/352) who may have good ovarian reserve and ovarian response. Several studies have confirmed that the reproduction of women decreases gradually but significantly starting approximately at 35 years and subsequently decreases more rapidly after 37 years[24][25].Female age is independent affected the oocyte quality and blastocyst formation,our data show that age does not affect the formation of useful blastocysts, which may be the enrolled patients had the better ovarian responsiveness .

The limitiation of the study is that it is retrospective and the sample is not enough big. Other clinical outcomes, such as live birth rate,fetal health.has not been observed in this study,expanded sample size is necessary to further verification.

\section{Conclusion}

Our study is to investigate the useful blastocyst formation rate in relation to bLH levels in IVF/ICSI cycles, showing that high bLH is associated with higher ovarian response and higher rates of useful blastocyst.AMH,bFSH and AFC which were defined as ovarian reserve and ovarian response is seem not association with the useful blastocyst formation.

\section{Abbreviations}

UBIVFC囚useful blastocyst in vitro formation capacity UBIVFR: useful blastocyst in vitro formation rate PCOS $\triangle$ Polycystic ovary syndrome DOR:ovarian function decline

$\mathrm{SD} \llbracket$ standard deviation BMI囚body mass index AFC囚the number of antral follicles

bLH囚the basal luteinizing hormone OSI囚ovarian sensitivity index 
HH囚hypogonadotropic hypogonadism

\section{Declarations}

\section{Acknowledgements}

None

\section{Authors'contributions}

Chun-Mei Yu is the main contributor to study design, data collection, statistical plan, data interpretation, and manuscript draft/revision. Xiu-Liang Dai participated in designing the study, data collection and interpretation, and manuscript revision. Yu-Feng Wang participated in data collection and critically revised the manuscript. Li Chen assisted with gynecological expertise in study design, data interpretation, and manuscript revision. All authors have read and approved the final manuscript.

\section{Funding}

This project is funded by basic \& applied research project of Changzhou

(CJ20179049) and special fund for clinical research of Chinese Medical Association. Xiu-Liang Dai of the funding resources participated in the study design, data collection, analyses, data interpretation, writing of the manuscript, or the decision to submit the manuscript for publication.Open access funding provided by Nan jing Medical University.

\section{Availability of data and materials}

The datasets used and/or analysed during the current study are available from the corresponding author on reasonable request.

\section{Ethics approval and consent to participate}

All procedures performed in studies involving human participants were in accordance with the ethical standards of the institutional and/or national research committee and with the 1964 Helsinki declaration and its later amendments or comparable ethical standards. The study protocol was approved by the Ethics Committee of the Changzhou maternal and child health care hospital(17020490718). Informed consent was obtained from individual participants included in the study. 


\section{Consent for publication}

Not applicable.

\section{Competing interests}

The authors declare that they have no competing interest.

\section{Author details}

Chun-Mei Yu, Xiu-Liang Dai, Yu-Feng Wang and Li Chen, Department of Reproductive Medicine Center, the Affiliated Changzhou Maternal and Child Health Care Hospital of Nanjing.

\section{References}

1. Gardner DK, Vella P, Lane M, Wagley L, Schlenker T, Schoolcraft WB. Culture and transfer of human blastocyts increases implantation rates and reduces the need for multiple embryo transfers. Fertility and Sterility 1998;69(1):84-8.

2. JOINT SOGC-CFAS.Guidelines for the number of embryos to transfer following in vitro fertilization No.182,September 2006[J].Int JGynaecol Obstet\2008囚102(2) : 203-216.

3. Gardner DK, Vella P, Lane M, Wagley L, Schlenker T, Schoolcraft WB.Culture and transfer of human blastocysts increases implantation rates and reduces the need for multiple embryo transfers. Fertil Steril 1998;69:84-8.

4. Veeck LL, Bodine R, Clarke RN, Berrios R, Libraro J, Moschini RM, et al. High pregnancy rates can be achieved after freezing and thawing human blastocysts. Fertil Steril 2004;82:1418-27

5.E. Fragouli S. Alfarawati, K. Spath, et al.Morphological and cytogenetic assessment of cleavage and blastocyst stage embryos.Molecular Human Reproduction2014;20:117-126

6. Stoop D, Ermini B, Polyzos NP, Haentjens P, de Vos M, Verheyen G, et al.Reproductive potential of a metaphase II oocyte retrieved after ovarian stimulation: an analysis of 23354 ICSI cycles. Hum Reprod 2012;27:2030-5.

7.Gardner DK, Schoolcraft WB. In virto culture of human blastocyst. In Janson R, Mortimer D (eds). Towards Reproductive Certainty: Infertility and Genetics Beyond 1999.Carnforth: Parthenon Press, 1999, 378-388.

8.Park, K.E., Ku, S.U., Jung, K.C., Liu, H.C., Kim, Y.Y., Kim, Y.J., Kim, S.H., Choi, Y.M., Kim, J.G. \& Moon, S.Y. (2013). Effects of urinary and recombinant gonadotropins on in vitro maturation outcomes of mouse preantral follicles. Reprod. Sci. 20, 909-16. 
9.Cortvrindt, R.G., Hu, Y., Liu, J. \& Smitz, J.E. (1998). Timed analysis of the nuclear maturation of oocytes in early preantral mouse follicle culture supplemented with recombinant gonadotropin. Fertil. Steril. 70, 1114-25.

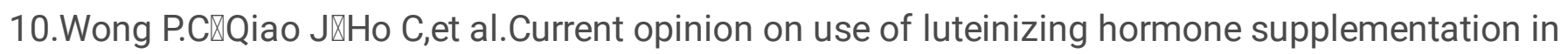
assisted reproduction therapy:an Asian perspective .Reprod Biomed Online,2011,23:81-90

11.Fleming R,Lioyd F,Herbert M,et al. Effected of profound suppression of luteinizing hormone during ovarian stimulation on follicular activity,oocyte and embryo function in cycles stimulated with purified follicle stimulating hormone.Hum Reprod,1998,13:1788-92

12.Balasch J,Vidal E,Penarrubia J,et al.Suppression of LH during ovarian stimulation:Analysing threshold values and effects on ovarian response and the outcome of assisted reproduction in the down-regulated women stimulated with recombinant FSH.Hum Reprod,2001,16:1636-43

13..Shoham Z.The clinical therapeutic window for luteinizing hormone in controlled ovarian stimulation.Fertil Steril囚2002,77ه1170-1177

14.Malin Huber, M.D., Nermin Hadziosmanovic, M.Sc., Lars Berglund, Ph.D., et al.Using the ovarian sensitivity index to define poor, normal, and high response after controlled ovarian hyperstimulation in the long gonadotropin-releasing hormone-agonist protocol:suggestions for a new principle to

solve an old problem.Fertility and Sterility.2013;11:1270-1276

15. Selcuk Selcuk, Bulent Emre Bilgic, Cetin Kilicci ,et.al.Comparison of ovarian responsiveness tests with outcome of assisted reproductive technology -a retrospective analysis.Arch Med Sci .2018;14, 851859

16. Esposito MA, Coutifaris C, Barnhart KT.A moderately elevated day $3 \mathrm{FSH}$ concentration has limited predictive value, especially in youngerwomen.HumReprod2002;17:118-23.

17.Steiner AZ, Herring AH, Kesner JS, et al.Antimüllerian hormone as a predictor of natural fecundability in women aged 30-42 years.Obstet Gynecol 2011;117:798-804

18. Broekmans FJ, Kwee J, Hendriks DJ,Mol BW, Lambalk CB. A systematic review of tests predicting ovarian reserve and IVF outcome. Hum Reprod Update 2006;12:685-718.

19.Frank Z. Stanczyk, PhD,James S. Kesner, PhD,Juliana W. Meadows, PhD.Association Between Biomarkers of Ovarian Reserve and Infertility Among Older Reproductive Age Women.JAMA.2017 (10) ; 318(14): 1367-1376.

20. Lauren A. Bishop M.D.Kevin S. Richter, Ph.D.George Patounakis M.D.Ph.D.Diminished ovarian reserve as measured by means of baseline follicle-stimulating hormone and antral follicle count is not 
associated with pregnancy loss in younger in vitro fertilization patients.Fertility and Sterility.2017(12);108(6):980-98

21. Practice Committee of the American Society for Reproductive Medicine. Testing and interpreting measures of ovarian reserve:a committee opinion. Fertil Steril 2015;103:e9-17.

22. Igarash $\mathrm{H}$, Takahashi T, Nagase $\mathrm{S}$. Oocyte aging underlies female reproductive aging: biological mechanisms and therapeutic strategies. Reprod Med Biol. 2015;14:159-169.

23.Sauer MV. Reproduction at an advanced maternal age and maternal health. Fertil Steril. 2015;103:1136-1143

24.Crawford NM, Steiner AZ. Age-related infertility. Obstet Gynecol Clin. 2015;42:15-25.

25.American College of Obstetricians and Gynecologists Committee on Gynecologic Practice and Practice Committee. Female age-related fertility decline. Committee Opinion No. 589. Fertil Steril.2014;101:633-634.

\section{Tables}

Table 1 Patient characteristics compared between two groups 


\begin{tabular}{|c|c|c|c|c|}
\hline \multirow{2}{*}{\multicolumn{2}{|c|}{ characteristic }} & GroupA & GroupB & P-value \\
\hline & & 354 & 352 & \\
\hline \multicolumn{2}{|l|}{ Ages(years) } & $30.1[4.0]$ & $29.8[4.0]$ & 0.46 \\
\hline \multicolumn{2}{|l|}{$\mathrm{BMI}(\mathrm{kg} / \mathrm{m} 2)$} & $23.1[4.7]$ & $22.8[4.2]$ & 0.75 \\
\hline \multicolumn{2}{|l|}{$\mathrm{AMH}$} & $5.25[4.19]$ & $5.12[2.98]$ & 0.62 \\
\hline \multicolumn{2}{|l|}{ Basic FSH } & $6.45[1.94]$ & $6.35[2.08]$ & 0.89 \\
\hline \multicolumn{2}{|l|}{$\mathrm{AFC}$} & $11.28[6.00]$ & $12.05[6.25]$ & 0.10 \\
\hline \multicolumn{2}{|c|}{ Duration of infertility } & $3.19[2.0]$ & $3.17[2.0]$ & 0.88 \\
\hline \multicolumn{2}{|l|}{ Basic LH } & $5.31[3.20]$ & $5.68[3.04]$ & 0.0006 \\
\hline Basic & E2 & $38.74[20.7]$ & $34.51[21.7]$ & 0.53 \\
\hline Basic & $P$ & $7.21[20.5]$ & $9.15[28.6]$ & 0.16 \\
\hline Basic & $\mathrm{T}$ & $24.26[14.86]$ & $23.81[15.57]$ & 0.54 \\
\hline \multicolumn{2}{|c|}{ Partner Ages $\square$ years $\square$} & $31.7[6.0]$ & $31.5[6.0]$ & 0.61 \\
\hline \multicolumn{2}{|c|}{ DFI } & $11.84[8.94]$ & $11.79[8.66]$ & 0.64 \\
\hline \multicolumn{2}{|c|}{ Partner BMI(kg/m2) } & $24.92[6.0]$ & $24.98[5.0]$ & 0.59 \\
\hline \multicolumn{5}{|c|}{ Diagnoses } \\
\hline \multicolumn{2}{|c|}{ Endometriosis } & $12 / 345$ & $18 / 345$ & 0.35 \\
\hline \multicolumn{2}{|c|}{ Male factor } & $30 / 345$ & $32 / 345$ & 0.89 \\
\hline \multicolumn{2}{|c|}{ Ovulation disorders } & $43 / 345$ & $37 / 345$ & 0.55 \\
\hline \multicolumn{2}{|c|}{ Tubal factor } & $223 / 345$ & $206 / 345$ & 0.21 \\
\hline \multicolumn{2}{|c|}{ Unexplained } & $34 / 345$ & $48 / 345$ & 0.13 \\
\hline \multicolumn{5}{|c|}{ Infertility type } \\
\hline \multicolumn{2}{|c|}{ Primary infertility } & $188 / 354$ & $172 / 352$ & 0.29 \\
\hline \multicolumn{2}{|c|}{ Secondary infertility } & $166 / 354$ & $180 / 352$ & 0.29 \\
\hline \multicolumn{5}{|c|}{ Ovulation promotion program } \\
\hline \multicolumn{2}{|c|}{ GnRH-a parogram } & $254 / 354$ & $272 / 352$ & 0.10 \\
\hline \multicolumn{2}{|c|}{ Others } & $100 / 354$ & $80 / 352$ & 0.10 \\
\hline
\end{tabular}

Table 2 The cycle characteristics of controlled ovarian stimulation in two regimens 


\begin{tabular}{|c|c|c|c|}
\hline characteristic & GroupA & GroupB & P-value \\
\hline & 354 & 352 & \\
\hline Gn dose (IU) & $1883[1275]$ & $1664[962]$ & 0.001 \\
\hline Gn duration $(d)$ & $8.96[2.0]$ & $8.68[1.0]$ & 0.07 \\
\hline$>14 \mathrm{~mm}$ follicles on hCG administration day (n) & $13.87[6.25]$ & $14.66[6.75]$ & 0.02 \\
\hline \multicolumn{4}{|l|}{ Trigger day } \\
\hline FSH(IU/L) & $12.02[7.52]$ & $10.84[5.42]$ & 0.02 \\
\hline $\mathrm{LH}(\mathrm{IU} / \mathrm{L})$ & $2.13[1.30]$ & $2.09[1.38]$ & 0.80 \\
\hline $\mathrm{E} 2(\mathrm{ng} / \mathrm{L})$ & $4078[2446]$ & $4450[2539]$ & 0.02 \\
\hline $\mathrm{P}(\mathrm{ng} / \mathrm{L})$ & $0.90[0.49]$ & $0.83[0.43]$ & 0.07 \\
\hline Oocytes retrieved & $12.20[4.25]$ & $12.87[5.0]$ & 0.008 \\
\hline MII oocytes (n) & $11.56[5.0]$ & $12.36[4.75]$ & 0.004 \\
\hline Normal Fertilized & $3308 / 4094 \square 80.8 \% \square$ & $3565 / 4349 \square 82.0 \% \square$ & 0.17 \\
\hline \multicolumn{4}{|l|}{ Oocytes rate $(\%)$} \\
\hline Top-quality embryosrate (\%) & $2200 / 3308 \square 66.5 \% \square$ & $2949 / 3565 \square 82.7 \% \square$ & $\square 0.0001$ \\
\hline FET clinical pregnancy rate & $\square 32 / 62 \square 51.6 \%$ & $\square 45 / 61 \square 73.7 \%$ & 0.01 \\
\hline
\end{tabular}

Table 3 The proportion of traditional ovarian reserve marker between two groups 


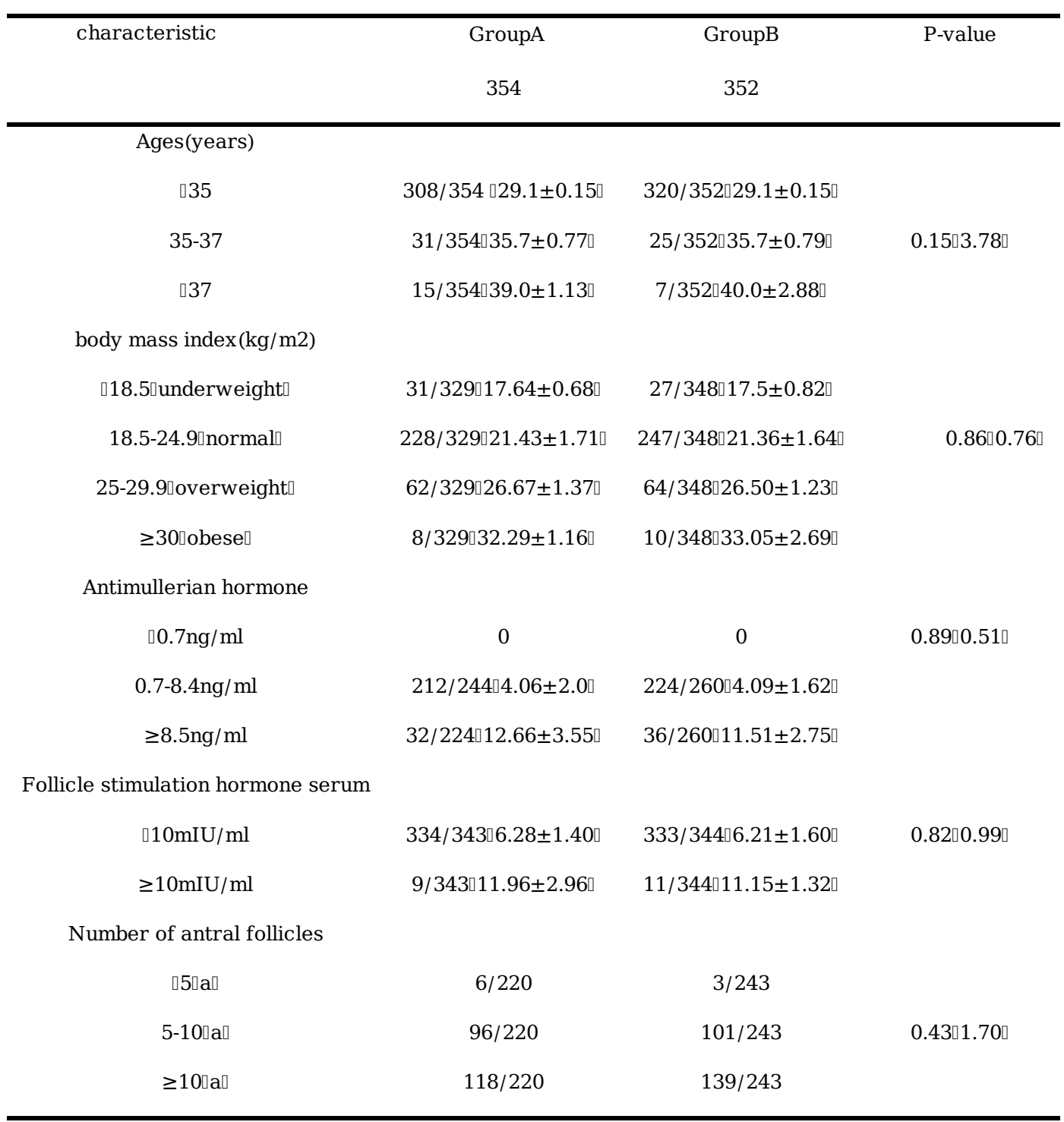

